\title{
Partial Discharge Signal De-noising Method Based on EMD and High Order PDE
}

\author{
Ganggang Zhao*, Fujiang Mo, Hui Chen, Peipei Wang and Mengsu Xu \\ School of Electrical Information Engineering, Jiangsu University, China \\ *Corresponding author
}

\begin{abstract}
The voltage level of power system is continuously improved. Partial discharge (PD) test is an important means to detect the insulation performance of high voltage electrical equipment. The electromagnetic environment of the test site is complicated. The extracted PD signal is submerged by noise. In order to get a more real PD signal, noise removal is inevitable. Aiming at this aspect, this paper proposes using empirical mode decomposition (EMD) and high order partial differential equation (PDE) to suppress the interference signal. The simulation results show that using this method to deal with the effect of PD signal is very obvious.
\end{abstract}

Keywords-partial discharge signal; EMD; PDE; de-nosing

\section{INTRODUCTION}

High voltage electrical equipment exists in the power transmission process of each link. Partial discharge test can effectively evaluate the insulation performance of high voltage electrical equipment [1]. At present, high voltage direct current transmission is a key research direction, and the research of DC partial discharge mechanism is still in its initial stage. At present, there is no obvious difference between AC and DC partial discharge detection methods. However, whether it is AC test, DC test, the test process will always be subjected to a variety of interference. Electronic switch action, pulse interference, white noise and so on everywhere [2], In this case, the PD signal has been unable to be recognized. Under normal circumstances, we can be simple to establish the following mathematical model to represent the interference of the PD signal:

$$
s(t)=p(t)+m(t)+n(t)
$$

here $p(t)$ is actual $\mathrm{PD}$ pulse; $m(t)$ is periodic interference pulse; $n(t)$ is white noise.

PD signal interference suppression process is to design an algorithm that makes the $p(t)$ separated from $s(t)$, remove the interference components. With the good effect of image processing by using higher order partial differential equation [3], we will use it to suppress the noise of PD signal.

\section{Mathematical Model Of Pd Signal}

PD signal is characterized in that the amplitude is small, usually for millivolt level; short duration, usually for a nanosecond. This paper use single exponential and double exponential decay models, can be expressed by formula (2), and shown in Figure I:

$$
p(t)=A\left(e^{-t / \tau_{d}}-e^{-t / \tau_{f}}\right) \sin \left(2 \pi f_{c} t\right)
$$

here $A$ is signal amplitude; $\tau_{d}$ and $\tau_{f}$ are time constants; $f_{c}$ is oscillation frequency.

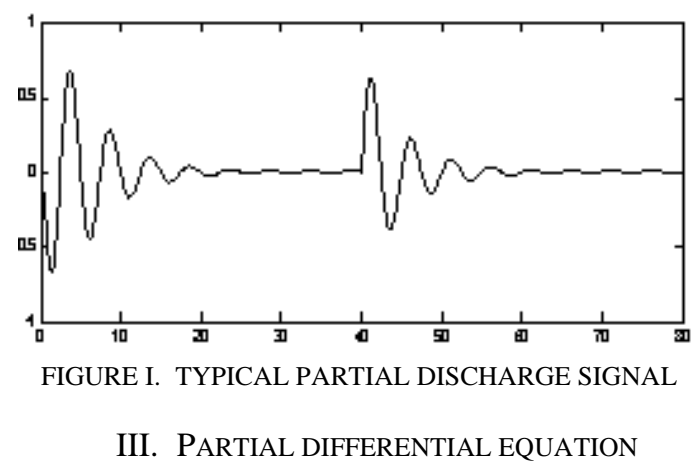

A. Image De-noising Model

Following the classical P-M anisotropic diffusion equation proposed by PERONA et al [4]. In 2000, the following four order partial differential equation was put forward by Yu-Li et al:

$$
\frac{\partial u}{\partial t}=-\nabla^{2}\left(c\left(\left|\nabla^{2} u\right|\right) \nabla^{2} u\right)
$$

here $\nabla$ is Hamilton Operator; $c(x)$ is a minus function. $c(0)=1$ and $c(\infty)=0$

Formula (3) has been widely used in image noise removal, as a classic image de-noising model, PERONA et al are given a typical representation of $c(x)$ :

$$
c(x)=\left(1+x^{2} / k^{2}\right)^{-1} \text { or } c(x)=e^{-x^{2} / k^{2}}
$$

here $k$ represent gradient threshold, generally take a very small value.

\section{B. Numerical Solution of the Equation}

Solution formula (4) corresponding to the four order partial differential equations, it will achieve the purpose of image de-noising [5]. Reducing the dimension of the equation and it 
can be used as the PD signal de-noising model. Hypothesis $y=0$, therefore $u(x, y, t)=u(x, 0, t)$ and abbreviated as $u(x, t)$. By means of numeration simulation of the equation, we can quickly calculate it based on MATLAB programming.

Suppose that $h$ and $\tau$ is the space step and time step. Order $x_{i}=i h, t_{n}=n \tau$, the grid is established with two parallel lines parallel to the $x$ axis and the $t$ axis. Based on the basic theory of calculus:

$$
\nabla^{2} u_{i}^{n}=\frac{1}{h^{2}}\left(u_{i+1}^{n}+u_{i-1}^{n}-2 u_{i}^{n}\right)
$$

Order $c\left(\left|\nabla^{2} u\right|\right) \nabla^{2} u=f\left(\nabla^{2} u\right)$ :

$$
f_{i}^{n}=f\left(\nabla^{2} u_{i}^{n}\right)
$$

On one hand:

$$
\nabla^{2} f_{i}^{n}=\frac{1}{h^{2}}\left(f_{i-1}^{n}+f_{i+1}^{n}-2 f_{i}^{n}\right)
$$

On the other hand:

$$
u_{i}^{n+1}=u_{i}^{n}-\tau \nabla^{2} f_{i}^{n}
$$

Simultaneous (5)(6)(7)(8), We get the final formula:

$$
\begin{aligned}
& u_{i}^{n+1}=u_{i}^{n}-\frac{\tau}{h^{2}}\left[f\left(\frac{u_{i}^{n}+u_{i-2}^{n}-2 u_{i-1}^{n}}{h^{2}}\right)\right. \\
& \left.+f\left(\frac{u_{i}^{n}+u_{i+2}^{n}-2 u_{i+1}^{n}}{h^{2}}\right)-2 f\left(\frac{u_{i+1}^{n}+u_{i-1}^{n}-2 u_{i}^{n}}{h^{2}}\right)\right]
\end{aligned}
$$

The PD signal with noise as the initial value, based on the recursive relation given by the formula, In turn to obtain $u_{i}^{1}, u_{i}^{2}, u_{i}^{3} \ldots .$. , after the appropriate number of iterations, we can restore the true PD signal.

\section{EMD AlgORITHM}

In recent years, many scholars have carried out extensive research on the EMD algorithm. Up to now, although there are some shortcomings of EMD algorithm [6], the mathematical theory is not perfect; there are some problems such as end effect, mode mixing and so on. But the algorithm in the application of the process has shown unparalleled superiority. Different from wavelet decomposition, The EMD algorithm divides the signal from high frequency to low frequency into a number of intrinsic mode function (IMF) and a residual component according to its own characteristics [7]. The result of decomposition is expressed by the following formula:

$$
s(t)=\sum_{i=1}^{n} I M F_{i}(t)+r_{n}(t)
$$

here $I M F_{i}(t)(i=1,2 \ldots n)$ are intrinsic mode functions. $r_{n}(t)$ is residual component.

Each IMF must meet the conditions [8]: (a) The number of extreme points and the number of zero crossing does not exceed 1; (b) At any point, the mean of the upper and lower envelope is 0 . General steps of empirical mode decomposition [9]:

(1). Find all the maxima and minima of the signal $s(t)$, then fit the maximum and minimum envelope $e_{+}(t)$ and $e_{-}(t)$ respectively, and get the average value of the upper and lower envelope, that is:

$$
m_{1}(t)=\left(e_{+}(t)+e_{-}(t)\right) / 2
$$

(2). Removal of the component $m_{1}(t)$ in the signal $s(t)$, then get the residual component $h_{1}(t)$, that is:

$$
h_{1}(t)=s(t)-h_{1}(t)
$$

Judge $h_{1}(t)$ whether to meet the IMF condition, if not satisfied, the $h_{1}(t)$ in accordance with the process of processing the $s(t)$ repeated processing, assuming that $k$ times after getting $h_{k}(t)$ to meet the IMF condition, then the first IMF component is $I M F_{1}(t)=h_{k}(t)$.

(3). Separating the first IMF component from $s(t)$ :

$$
r_{1}(t)=s(t)-F_{1}(t)
$$

(4). Regard $r_{1}(t)$ as $s(t)$ of (1), repeat use (1) - (3) steps, Separate out $F_{2}(t)$, repeat process all above, until the signal $r_{n}(t)$ appears monotonic or constant after $n$ times decomposition, the end of the decomposition.

\section{PROCESS OF PD SIGNAL DE-NOSING}

In this paper, combined with the high order PDE and EMD two algorithms, the specific algorithm steps are:

(1) Using the EMD algorithm to decompose the signal, the results of the decomposition is the formula (10);

(2) Using four orders PDE to various IMF components for iterative de-noising;

(3) Reconstruction of these signals, we can get PD waveform.

It is worth noting that the whole process is not complicated, but there are many details of the problem. For example: in the EMD decomposition process often encounters end effects, mode mixing and other issues, Many scholars put forward the corresponding methods can suppress these problems to a certain extent, but this is not the focus of this paper. Another important question is how to get the appropriate number of iterations to ensure the speed of calculation. EMD algorithm 
decomposes the signal from high frequency to low frequency. In the high frequency, more iterative times are used. This section describes the process of PD signal interference suppression, the next section, we will use a simple simulation shows the effect of this approach.

\section{Simulation Of Pd Signal De-Nosing}

\section{A. Noise of PD Signal}

Partial discharge signal is a kind of weak signal. And it is easy to be covered by all kinds of noise [10]. the main component of noise is white noise signal and narrow band cycle signal. Due to the existence of this interference, makes the PD signal can not be used as the basis for partial discharge test. Formula (1) has been given a partial discharge signal mathematical model, according to the mathematical model; we can use MATLAB to simulate the PD signal containing noise. Here the periodic interference signal $m(t)$ takes a simple sinusoidal signal; In addition, generating a white noise signal, the two signals is added together to the signal $p(t)$, the simulation waveform as shown in Figure II.

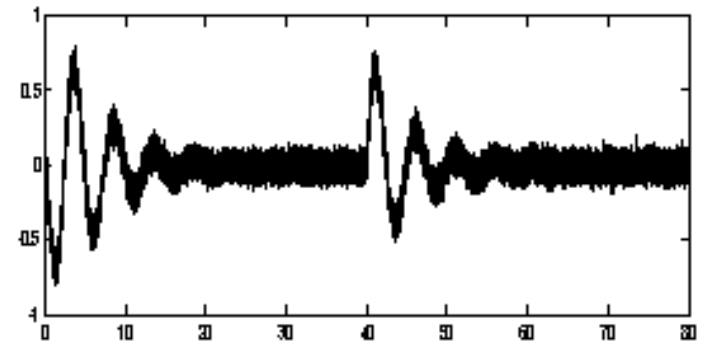

FIGURE II. PARTIAL DISCHARGE SIGNAL WITH NOISE

\section{B. Simulation Results}

In order to show that the method proposed in this paper can achieve a better effect on the interference suppression of partial discharge signals, this paper chooses to use MATLAB to program the algorithm. The effect of de-noising is shown in Figure III.

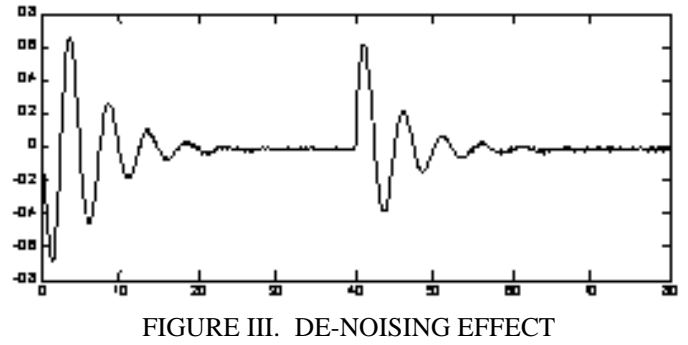

\section{CONCLUSIONS}

For the convenience of observation, the partial discharge signal of the simulation and the partial discharge signal after de-noising are drawn in the same picture, as shown in Figure IV, red and black, respectively, on behalf of the original waveform and waveform after de-nosing.

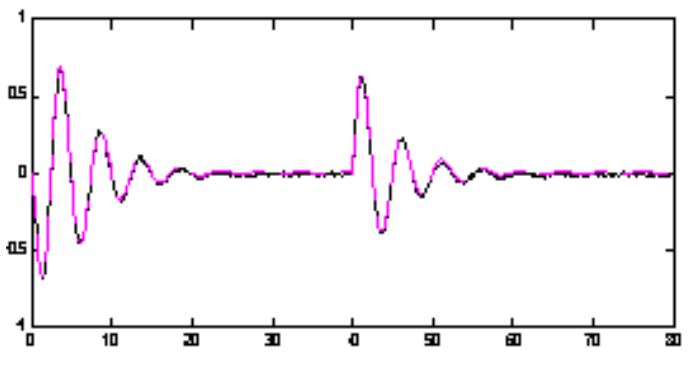

FIGURE IV. WAVEFORM COMPARISON

After simulation, we can draw the conclusion that the effect is very obvious when combined with the EMD algorithm and the high order partial differential equation to deal with the PD signal. It can be seen that the method can effectively preserve the edge information of the signal from the PD signal waveform. After the experiment learned that in the high order partial differential equations iterative de-noising process, the number of iterations is not the more the better. Experimental results show that with increasing the number of iterations, signal-to-noise ratio increased first and then decreased trend. According to the experience, different noise characteristics need different iteration times, the choice of the appropriate number of iterations need combine the experience and the calculation, can be used as the focus of the next step.

\section{ACKNOWLEDGMENT}

Firstly, I would like to show my deepest gratitude to my teacher, Dr. Mo, a respectable and talented man, who has provided me with valuable guidance in every stage of the writing of this thesis. Without his enlightening instruction, impressive kindness and patience, I could not have completed this thesis. Secondly, I would like to thank the topic group of the other students to give me advice, they support me to complete this article.

\section{REFERENCES}

[1] J Liu, G Zhang et al. "Study on Miniaturized UHF Antennas for Partial Discharge Detection in High-Voltage Electrical Equipment” .Sensors.2015,15(11):29434-29451.

[2] H-Li, H Peng et al. "Study on Suppressing White Noises and Periodic Narrow Bandwidth Noise of PD by Vertically Invariant Gray-Level Morphological Filter”. International Conference on E-product E-service \& E-entertainment.2010:1-3.

[3] X Liu, L Huang. "Adaptive fourth-order partial differential equation filter for image denoising”. Applied Mathematics Letters.2011,24(8):1282-1288.

[4] S Hu. "External Fractional-Order Gradient Vector Perona-Mlik Diffusion for Sinogram Restoration of Low-Dosed X-Ray Computed Tomography”. Advances in Mathematical Physics.2013(6):757-786.

[5] L Zhao, A Yin. "High-order partial differential equation de-noising method for vibration signal”. Mathematical Methods in the Applied Sciences.2015,38(5):937-947.

[6] P Chen, Y Lu. "Research on Fault Diagnosis Method of Rolling Bearing Based on EEMD and Hilbert". Electric Power Science \& Engineering.2013,9.

[7] T Ghosh, D Bhattacharyya et al. "A Review on Different Techniques to De-noise a Signal”. International Journal of Control \& Automation.2014,7.

[8] Y Wang, Y Liu et al. "Signal prediction based on empirical mode decomposition and artificial neural networks". Geodesy \& Geodynamics.2012,3(1):52-56. 
[9] JR Yeh, TY Lin et al. “Applying Similarity Theory and Hilbert Huang Transform for Estimating the Differences of Pig's Blood Pressure Signals between Situations of Intestinal Artery Blocking and Unblocking”. Enformatika. 2007(1-4):468.

[10] P Drexler, M Cap et al. "Detection of low-level electromagnetic signal of partial discharge by means ofdisturbed acquisition discrimination". Piers Proceedings.2014. 